\title{
The Preventive Strategy of TV News in the New Media Age
}

\section{Feifei Jing}

\section{Weinan Teacher's College, Weinan 714099, China}

\begin{abstract}
With the continuous development of network technology and information transmission, the new media age with mobile phone and Internet as the main part has come, which make the way that people receive information has changed a lot. Competition between we media platform is becoming increasingly fierce. In such circumstances, people access to information channels are no longer confined to television news, which shortens the time people touch television news, but also divert a large number of young audience of the TV news. For a better respond to the challenges brought by the new media age and defending the status of mainstream media, television news programs need to transform to meet the needs of the audience better and conform to the development of the age. Only in this way can we guarantee our own position.
\end{abstract}

Keyword: New media age; Television news; Status; Preventive strategy

\section{新媒体视域下电视新闻的应对策略}

\author{
敬菲菲 \\ (渭南师范学院, 陕西渭南 714099)
}

摘要: 随着网络技术以及信息传播方式的不断发展, 以手机和互联网为主体的新媒体时代已经到来, 这使得人们接收信 息的方式发生了非常大的改变，自媒体平台的竞争日渐激烈。在这样的大环境下，人们获取信息的渠道不再仅仅局限于电视 新闻, 这缩短了人们接触电视新闻的时间, 更分流了电视新闻一大批年轻的观众。为了更好的应对新媒体时代带来的挑战, 捍卫主流媒体的地位, 电视新闻类节目需要转型, 更好的迎合观众的需求, 顺应时代的发展。只有这样才能够保证我们自身 地位。

关键词: 新媒体时代; 电视新闻; 现状; 应对策略

引言

新媒体时代的到来使得各种传播媒体之间的竞争日益激励, 为了能够站稳市场, 很多媒体平台可以说 是 “殚精竭虑”。相比较自媒体平台的崛起, 新媒体时代的到来却使得传统电视媒体在传媒行业中的地位 一直在不断下降，其实这也表明电视新闻在当今确实有其不足之处。下文从新媒体时代的特征入手，分析 了新媒体时代的到来对电视新闻到来的影响, 并且对电视新闻如何应对新媒体时代带来的挑战做出了自己 的总结。

\section{1 新媒体时代的基本概述}

新媒体是相对于传统媒体而存在的，指的是利用数字技术、互联网技术等新兴技术，借助于手机、电 脑等终端向用户提供一种交互式信息的传播服务。而新媒体时代的到来则是指的当下这种传播服务已经占 据了这个时代的主流, 越来越多的人生活中已经离不开这种信息传播服务。“11相比较传统电视新闻媒体, 新媒体时代更具开放性、交互性以及时效性, 处在信息接收方的用户可以自由 “定制” 自己所需要的信息, 最大限度的满足他们的个人喜好。从另一个角度来说, 信息传播数量的增加其实也扩大了新媒体的接受人 群, 这也是其受众不断增多的一个非常重要的原因。

根据相关数据的统计, 我国网民数量已然突破七亿大关, 互联网普及率已经突破百分之五十, 其中通 
过手机上网的用户已经占到了总体网民数量的百分之九十。人们接受信息的方式在改变的同时，电视新闻 节目的观众也在逐渐被分流。很多电视新闻节目的观众已经呈现了老龄化的趋势, 90 后甚至是 80 后人群 更倾向于享受新媒体时代所带来的信息传播方式。这样的现状必然会导致电视新闻节目的收视率不断下 降, 广告收入大幅度减少, 这类问题甚至已经威胁到了一些地方台的生存。面对如此严峻的局面, 电视新 闻节目只有进行全方位的调整才能够适应社会发展的节奏。

\section{2 新媒体时代对电视新闻的影响}

\section{1 积极影响}

首先新媒体时代使得电视新闻的内容开始变的多元化。这是因为新媒体时代出现了大量的自媒体平 台, 并且这类自媒体平台的热度一度超过了主流电视媒体。电视新闻节目想要生存只能去借鉴他们的优势, 这对电视新闻节目也是一个鞭策。而新闻节目的内容也逐渐多样化，像如财经新闻、时政新闻以及民生新 闻已经占据了很多电视台的半壁江山。这从另一个角度同样体现了新媒体时代下，电视新闻节目的制作正 在趋向于精品化。 ${ }^{\mathbf{2} 21}$ 此外很多新闻节目在直播的过程中也开始利用微博、网上论坛以及现场连线的形式让 观众参与到直播的过程中, 与观众零距离交流。所以说现在的电视新闻节目从某些方面来说具备了自媒体 平台的优势, 观众也可以参与到直播的过程中, 也可以对报道的内容自己进行评述，这不仅借鉴了新媒体 的运作模式, 更是依靠新媒体时代下相关的技术支持才能够完成的。最后新媒体时代, 各项新技术的应用 强有力的推动了电视新闻制作品质的提升, 新闻的采集、剪辑以及传输不管是速度还是质量上与过去相比 都有了很大的改变。时代在进步, 电视新闻也一直在适应时代的步伐。

\section{2 消极影响}

新媒体能够在短时间内将信息传递到每个人的手上，这样的传递几乎是在每天 24 小时不间断的进行, 这是传统电视媒体所不具备的。而传统的电视新闻可能对于一个事件的报道要固定在一个特有的时间段, 也就是节目播出的时间, 故此这种滞后性在新媒体时代将使得电视新闻的处境会变的更加艰难。其次当前 经济高速发展, 人们的价值观以及对于一些事物的认知也发生了不小的变化, 很多时候人们放弃电视新闻 只是因为自己内心的诉求并没有一个合理的宣泄渠道, 或许只是因为生活中的一件小事, 他们需要外界的 关注。 ${ }^{131}$ 而电视新闻是没有办法去 “迎合, 每一个人的价值观的, 它必须起到一个與论导向的作用, 但自 媒体平台因为其独有的特点却可以做到这一点。当然如果过分的去迎合就失去了媒体平台原本的意义, 也 会带来一系列的社会问题, 这就牵扯到新媒体时代对于新闻媒体行业的监管问题。但是站在观众的角度来 说, 我们应该认识到电视新闻正在逐渐走向落寞。

\section{3 电视新闻应对新媒体时代的具体措施}

\section{1 深入挖掘自身优势, 提高节目的竞争力}

虽然说新媒体时代的到来使得传统媒体的地位在不断下降，但是传统媒体也并不是没有可以值得新媒 体借鉴的优势。权威性、专业性、公信力以及影响力都是传统媒体的优势, 这一点对于电视新闻来说非常 重要。在新媒体时代, 电视新闻应该抓住这些优势提升节目的竞争力, 具体来说可以分为以下几个部分来 完成。

首先在日常的工作之中电视新闻从业者要有意识的去维护自身的公信力以及权威性, 这是因为虽然新 媒体信息的传播速度较快, 但是很多内容都是匿名发布, 所以说新闻信息的真实性就很难得到确认。 ${ }^{[4}{ }^{1}$ 此 
外当下网络上的民生新闻经常会遭受各种 “不友好” 的评价, 而且这些新闻观点本身就与当前社会主流价 值观相抵触, 所以说根本无法承担起正确引导社会與论, 权威解释以及维护社会稳定的作用。与之相对应 的, 电视新闻节目的制作就正规多了, 信息的采集、制作以及之后的编排和后期的播出都有严格的审查制 度, 所以说电视新闻节目的公信力在民众之中是有目共睹的。这就是在一些重大新闻事件的发布上, 公众 信赖电视新闻节目的原因。

其次推进电视新闻节目直播也是电视新闻挖掘自身优势, 应对新媒体时代的重要措施。直播是电视新 闻节目体现价值的最高手段, 也是信息传播的最终形态。推广直播这种节目播出方式是电视新闻与新媒体 竞争的重要法宝, 这是因为网络直播与电视新闻直播完全不可相比较, 两者传递的内容以及价值观有着明 显的差距。所以说今后, 新闻节目进行直播, 尤其是对重大事件进行直播报道, 将能够影响到当前社会主 流价值观, 又何惧新媒体的威胁呢。 ${ }^{51}$ 进行直播肯定少不了必要的设备投入以及建立起一支高素质的新闻 采编队伍, 虽然说现在很多节目组都购置了卫星直播车, 也在重视这方面人才的培养, 但是对于一些新闻 事件还是做不到及时有效的报道。当然这部分工作的成绩也是值得我们骄傲的, “汶川地震” 电视新闻直 播对救灾工作进行了全天候的报道, 全中国的目光通过深入灾区记者的镜头都汇集到了震区, 时时刻刻感 受着震区的每一点变化。未来作为电视新闻人, 我们一定要朝着这个方面继续去努力。

最后要注意的是, 我们要深入挖掘新闻的内涵。虽然说新媒体对于事件的传播速度非常快, 借助于网 络而且造成的影响也会比电视新闻大。但是这种传播仅仅只停留在新闻事件的表面, 没有进行深入的挖掘, 发现新闻事件背后的意义, 也不能满足公众深入了解新闻事件的诉求。 ${ }^{\mathbf{6} 1}$ 而电视新闻节目之中专业化的制 作团队, 就能够很好的满足挖掘新闻内涵的实际需要, 在进行新闻报道的基础之上, 对新闻事件进行纵向 以及横向的评述, 这能够帮助听众挖掘新闻事件背后的价值和意义。综合实际情况我们可以发现, 近年来 新闻评述以及深度报道类的节目非常容易受到听众的欢迎, 因此这也是提高节目竞争力的一个重要手段。

\section{2 融合互联网的优势}

融合互联网的优势主要是指电视新闻向互联网, 向自媒体学习的过程, 具体来说可以分为以下几个部 分。首先, 借助互联网, 加强传播的透明度。随着科学技术的发展, 互联网给我们的生活带来了翻天覆地 的变化, 也为各行各业的发展注入了活力。网络新闻之所以受到年轻人的喜爱和追捧, 除了它区别于纸质 或者视频的传统形式, 阅读新闻更加方便快捷之外, 更多的就是参与性, 它非常贴近群众的生活。网络新 闻中由于网友可以评论自己的观点, 使得网民也可以真正的参与进去, 这是电视新闻急需改进的地方。今 后电视新闻节目应该避免出现喊口号、讲空话的现象, 真正地从人们的生活里去发现民生问题, 解决群众 所需, 报道老百姓最关心的话题, 提高群众参与度, 加强新闻的透明度。电视新闻应该学习互联网新闻的 优点, 同时也要避免出现一些问题和漏洞, 由于网络时代信息的复杂性, 应该更多的关注信息的选择和篎 选，为人民群众提供更多真实健康的新闻消息。

其次, 加强與论监督, 引领社会焦点。综合各方面的数据显示, 电视新闻具有优势的新闻类型是时政 类, 例如国家召开的重大会议、法律政策或者提案的研读, 这也从侧面反映了电视新闻具有很高的权威性, 但是反过来讲他们一定程度上也 “不自觉” 地屏蔽了一些负面却真实存在的内容。 ${ }^{\mathbf{1} 71}$ 而网络新闻则恰恰相 反, 网络具有开放性和灵敏性的特点, 群众的参与度相对更高。像是我们曾经讨论的 “老人摔倒了扶不扶” 这样的热点话题, 也是网络上 “炒热”之后新闻媒体才开始报道的。因此新闻媒体应该保持自己的权威性 
和严肃性的优势, 学习网络新闻的敏感性, 关注更多的时政热点以及民生问题, 加强與论的监督, 引领社 会新闻的焦点。

最后，需要强调的就是加强对于民间新闻的整合力度。传统电视新闻的生产是相对封闭的，为了保证 文章的真实性, 多是出去跟踪报道或实地采访。现如今记者出去采访的相机、录音笔和录像的设备已经开 始走入普通家庭, 这也使得电视新闻逐渐丧失了这个优势。 ${ }^{\mathbf{1} 1}$ 不少的网民会利用这些设备将所拍的视频上 传到网络上, 但是忽视了其中的尺度, 一度也造成了网络新闻的混乱。 ${ }^{191}$ 电视新闻可以采纳群众所上传的 新闻消息和视频, 但是一定要注意内容的笁选和尺度的把握, 不断开发其中的潜在资源, 拓展自己的新闻 市场, 也借助网络的形式创新电视新闻的形式和内容, 为其增添新的色彩, 在新媒体时代下打好这场 “翻 身仗”。

\section{结论}

我国是一个具有 13 亿人口的大国, 所以说民众的與论是一股不可忽视的力量。而电视新闻就承担着 维护公众知情权以及稳定社会與论的重要作用。但是新媒体时代的到来使得传统电视媒体受到了不小的冲 击, 以电视新闻节目为例, 近几年就有大批的观众被分流到了新媒体之上。面对这样的局面很多电视新闻 节目都在进行调整, 以适应这一行业的发展现状。但是因为缺少具体的工作方案以及应对措施, 所以这部 分工作的效果并不尽如人意。如果单纯的把新闻中播出的内容, 打包之后放到自媒体平台之上, 是没有办 法 “拉回” 观众的。具体这部分的工作该如何开展, 需要我们综合当下的实际情况形成具体的工作方案。 上文是根据笔者的经验对新媒体时代电视新闻的应对策略进行的总结, 希望能够对改善当前电视新闻的工 作局面有所帮助。

\section{[参考文献]}

[1] 田伟茹. 新媒体时代电视新闻采编策略分析 [J]. 新闻传播, 2016, (16) :105-106.

[2] 赵亮. 刍议新媒体时代电视新闻采编策略 [J]. 新闻研究导刊, 2016, (08) :256.

[3] 吕宁. 新媒体时代电视新闻的创新发展策略 [J]. 新媒体研究, 2016, (06) :66-67.

[4] 张政. 新媒体时代电视新闻采编策略探析 [J]. 新闻研究导刊, 2016, (04) :229.

[5] 沈益顺. 自媒体时代电视新闻传播内容面临的挑战与应对 [J]. 新闻研究导刊, 2015, (20):219.

[6] 陈菊香. 新媒体时代电视新闻节目的应对策略 [J]. 西部广播电视, 2015, (10) :9-10.

[7] 黄小伟. 新媒体时代电视新闻采编策略分析 [J]. 中国报业, 2015, (04) :55-56.

[8] 张瑛. 新媒体时代电视新闻的应对策略研究 [J]. 科技传播, 2014, (23) : 84+88.

[9] 陈婧. 新媒体时代电视新闻面临的挑战与发展策略[J]. 视听, 2013, (09) :54-55.

\section{References}

[1] Tian Weiru Analysis on the Strategy of TV News Editing in the New Media Age [J] Journalism and Communication 2016,(16):105-106 (in Chinese)

[2] Zhao Liang A Rustic Opinion on the Strategy of TV News Editing in the New Media Age [J]. News Research Guide 2016,(08):256(in Chinese)

[3] Innovative Development Strategy of TV News in the New Media Age [J]. New Media Research2016,(06):66-67(in Chinese)

[4] Zhang zheng An Analysis on the Strategy of TV News Collection and Publication in the New Media [J]. Journalism Research Guide2016,(04):229(in Chinese) 
[5] Shen Yishun Challenges and Countermeasures of TV News Dissemination in Media Times [J] Journalism Research Guide,2015,(20):219(in Chinese)

[6] Chen Juxiang The Strategy of TV News Program in the New Media Age[J] Western Radio and Television ,2015,(10):9-10(in Chinese)

[7] Huang Xiaowei Analysis on the Strategy of TV News Editing in the New Media Age [J]. China Newspaper Industry, 2015,(04):55-56(in Chinese)

[8] Zhang Ying Research on the Strategy of TV News in the New Media Age[J].Science and Technology Communication 2014,(23):84-88(in Chinese)

[9] Chen Jing Challenges and Development Strategy of TV News in the New Media Age [J]. Audiovisual,2013,(09):54-55(in Chinese) 